\title{
Fracture propagation in DWT back slotted specimens
}

\author{
A. FONZO ${ }^{1}$, P. SALVINI ${ }^{1}$ and M. DI BIAGIO ${ }^{2}$ \\ ${ }^{1}$ University of Rome 'Tor Vergata', Dept. of Mechanical Engineering, Via del Politecnico n. 1, 00133 Rome \\ ITALY, (Fax: +39-06-2021351) \\ ${ }^{2}$ Centro Sviluppo Materiali S.p.A., Via di Castel Romano n. 100, 00128 Rome, ITALY, (Fax: +39-06-5055452) \\ E-mail addresses: fonzo@ing.uniroma2.it (A.Fonzo), salvini@uniroma2.it (P.Salvini),m.dibiagio@c-s-m-it \\ (M. Di Biagio)
}

\begin{abstract}
The present paper deals with the measurements of the critical Crack Tip Opening Angle by using an instrumented test. There is a comparison between the analysis of results made by a well known kinematic model and those given by finite element analysis based on a cohesive model for node release. Analysis is performed both on simple specimens and on back slotted ones.
\end{abstract}

Key words: Finite element simulation, drop weight tear test, ductile fracture.

\section{Introduction}

The present trend in gas delivery by pipelines at high pressure demands appropriate investigations into pipelines' behaviour when extraordinary events, such as fracture propagation, occur. For many years researchers have sought appropriate material characterization able to provide information on the pipe by means of simplified laboratory tests (Demofonti et al., 1995). Full thickness SENB specimens are often used to check the capability of the pipeline steel, so that a minimum fracture resistance is defined to prevent the stable propagation of a ductile fracture.

The Drop Weight Tear Test (DWTT) measures the whole energy consumed to completely fracture a notched specimen. One of its main advantages is the possibility to manage quite lengthy ligaments, so that almost stationary propagation is achieved during the test. With the aim of carrying out a reliable Finite Element analysis of a pipeline subjected to fracture phenomena, using an ad hoc developed code, (Berardo et al., 2000; Salvini et al., 1999, 2000; Fonzo et al., 2002), it is necessary for numerical simulations to be performed on DWT tests as well. These preliminary analyses are an essential step to 'tune up' fracture parameters that are used on a full burst test simulation. A critical point of Finite Element simulation regards the treatment of the zone surrounding the running crack tip, where fracture energy is progressively released. Among the several approaches that can be found, here a one-dimensional cohesive layer at crack flanks is assumed. This modelling option allows the gradual release of the constraints applied on nodes along the crack path. A softening zone is considered where internal energy is released for each node of the mesh: a progressive change drives from a full constrained condition to a complete released one. The model implemented requires the estimate of two fracture parameters: critical value of Crack Tip Opening Angle $\left(\mathrm{CTOA}_{\mathrm{C}}\right)$ and length of the so-called Fracture Process Zone where softening occurs $(\Delta)$ (Salvini et al., 2003; Cotterel et al., 1977). 


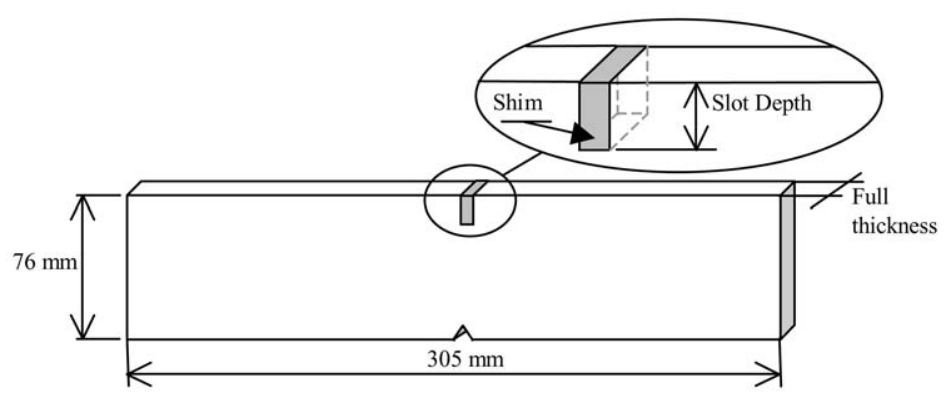

Figure 1. Scheme of Back Slot DWTT.

Although specimens are manufactured with the aim of evaluating stable ductile fracture propagations, we must not conceal the effects of real applications of boundary conditions: notch manufacturing, the interaction between plastic zones induced by an impacting hammer and by crack stresses ahead of the crack tip.

Back Slotted specimens offer an interesting opportunity to try to manage these undesirable effects, (Rudland et al., 2002); these specimens, schematized in Figure 1, prevent the crack from propagating until the back side of the specimen. Changing the depth of the back slotted shim offers an opportunity to directly measure the fracture energy released and, in the present paper, both test results and numerical simulations are compared each other. As a result, the region where stable crack propagation occurs is clearly defined, cleaning many polluting effects. A deeper comparison between experimental and numerical data gives a rigorous procedure for determination of the two fracture parameters required by the present model.

\section{Analysis of DWT test conditions}

The tests have been performed on back slot specimens with different slot depths. Non-back slot specimens have also been considered. The numerical approach requires a cohesive model for node release, which is described above; the kinematic model, providing an analytical solution based on geometrical and kinematic considerations, is also briefly described in the following section.

\subsection{The FInIte ELEMENT CODE DEVELOPED: PICPRO}

The FE program used in this work is named PICPRO and has been developed by the University of Rome 'Tor Vergata' in collaboration with the Centro Sviluppo Materiali S.p.A.

PICPRO is an explicit solutor based on the method of central differences and is, on purpose, designed to simulate the ductile fracture propagation in both large (e.g. pipelines) and small structures (like SENB specimens). Many algorithms capable of governing the modalities of crack advance are implemented within the code. In the case of a DWT Test, fracture can be managed by the same kinematic law hereinafter described, a Free fracture Propagation Algorithm (FPA). The first method allows the calculation of the crack speed by a simple equation outlined by Martinelli et al. (1996); the second one allows a more generic analysis of the fracture phenomenon and can be successfully used to simulate propagation both in stable and in transient conditions (Fonzo et al., 2002). In the FPA algorithm, during code execution, a step by step check on critical conditions is performed: when the actual fracture-characterizing parameter (e.g. $\mathrm{CTOA}_{A}$ ) is greater or equal to its critical value (e.g. $\mathrm{CTOA}_{C}$ ), crack advance 
can take place. There is still a wide discussion (Newman et al., 2003) about whether CTOA $_{C}$ is a material characteristic that can be effectively measured; in any case, thickness plays an important role, so all tests should be performed on full thickness. Another issue is the fact that $\mathrm{CTOA}_{\mathrm{C}}$ represents stable propagation; therefore, during non-stable propagation its value may change significantly.

The cohesive model implemented in PICPRO needs the determination of two parameters: $\mathrm{CTOA}_{\mathrm{C}}$ and $\Delta$, (Salvini et al., 2001). CTOA represents a very interesting parameter since is directly related to the geometrical aspect of the specimen in the region near the crack tip. According to many researchers it can be used for evaluating critical material conditions for crack propagation (Azodi et al., 1990; Rice et al., 1978; Wang et al., 1994). CTOA is the angle emerging from the opening crack flanks. With $\mathrm{x}$ the distance from the crack tip, oriented towards crack propagation, CTOA can be found by the limit applied to the incremental increase of the Crack Opening Displacement (COD). The actual value of the Crack Tip Opening angle $\left(\mathrm{CTOA}_{\mathrm{A}}\right.$ is given by:

$$
\mathrm{CTOA}_{\mathrm{A}}=\lim _{x \rightarrow 0^{-}}\left\{2 \cdot \arctan \left[\frac{1}{2} \frac{\Delta}{\Delta A} \operatorname{COD}(x)\right]\right\}
$$

Its critical value $\left(\mathrm{CTOA}_{\mathrm{C}}\right)$, used by PICPRO for the step by step comparison, is evaluated by experimental results of a DWT Test, (Salvini et al. (2003)), as shown in the following section. Hence the critical value of CTOA comes out from an elaboration of experimental results; on the other hand the dimension of the Fracture Process Zone $(\Delta)$, being directly responsible for the amount of energy consumption, can be easily estimated by varying its value until a good accordance between experimental and FE results is achieved during crack evolution (Salvini et al., 1999). For this second identification the attention is usually focused on load force (measured in correspondence of the impacting hammer) and time, these being the data directly collected during the test.

In this paper an accordance has been pursued also for impact load vs. crack length so that instant crack speed is also directly accounted for. Unfortunately, direct measurements of crack tip position are not a simple and inexpensive task. Nevertheless, the kinematic model can provide this information, by simple analytical manipulation of experimental data.

\subsection{THE KINEMATIC MODEL}

Martinelli et al. (1996) proposed a useful geometrical formulation, valid for SENB specimens, which allows the determination of $\mathrm{CTOA}_{\mathrm{C}}$ during propagation, based upon these hypotheses:

1. The crack tip opening angle keeps constant during whole propagation

2. The specimen rotates on a moving centre located at a distance $r^{*}(W-a)$ from the crack tip

3. The specimen rotates rigidly around a plastic hinge; this is equivalent to considering the residual ligament fully plasticized.

The parameter $r^{*}$ represents the ratio between the distance of instantaneous centre of rotation from crack tip with respect to residual ligament length. Its value is kept constant and equal to 0.45 , Matsoukas et al. (1984). These hypotheses allow the expression of the instantaneous crack growth as a function of kinematic parameters and CTOA: 


$$
\mathrm{d} a=\frac{r^{*}(W-a) \cdot \cos \left(\frac{\mathrm{CTOA}}{2}-\alpha_{p}\right)}{\sin \left(\frac{\mathrm{CTOA}}{2}\right) \cdot \cos \left(\alpha_{p}\right)} \cdot \mathrm{d} \alpha_{p},
$$

where $a$ is the crack length, $W$ the specimen width and $\alpha_{p}$ the plastic component of the rigidplastic half-specimen rotation angle computed from fracture initiation. Considering the first hypothesis and integrating Equation 2, crack length can be calculated as:

$$
a=W-(W-a) \cdot \cos \left(\alpha_{p}\right)^{r^{*}} \cdot \exp \left[-\frac{r^{*} \alpha_{p}}{\operatorname{tg}\left(\frac{\mathrm{CTOA}}{2}\right)}\right]
$$

The total rotation angle $\alpha$ can be expressed as a function of the impacting hammer displacement $s_{\text {HAMMER }}$ as shown in the following expression:

$$
s_{\text {HAMMER }} \approx \frac{\operatorname{span}}{2} \operatorname{tg}(\alpha)-\left(W-R_{\text {Hammer }}-R_{\text {support }}\right) \cdot \frac{1-\cos (\alpha)}{\cos (\alpha)},
$$

where span is the distance between supports, $R_{\text {HAMMER }}$ and $R_{\text {SUPPORT }}$ are, respectively, the radius of hammer and supports. The hammer displacement can be deduced, knowing the hammer mass and load force, integrating twice the well-known Newton's law.

Evaluation of the critical value of CTOA can be carried out based upon energy considerations that allow the correlation of the fracture energy propagation with CTOA (Martinelli et al., 1996).

$$
E_{P}^{a i \rightarrow a f}=\frac{\operatorname{tg}(\mathrm{CTOA})}{2 r^{*}} \cdot A^{*} \sigma_{0} B \cdot \int_{a i}^{a f} \frac{W-a}{1+\operatorname{tg}\left(\alpha_{p}\right) \operatorname{tg}\left(\frac{\mathrm{CTOA}}{2}\right)} \mathrm{d} a
$$

In Equation 5, $A^{*}$ is a constant (assumed value is 1/3, Knott, 1973), that takes into account the real stress distribution in the plastic hinge; $B$ is the specimen thickness, $E_{P}^{a i \rightarrow a f}$ represents the energy related to fracture propagation that is dissipated for a crack length increment from an initial value $(a i)$ to a final value $(a f)$. Initiation energy is not taken into account. The values $a i$ and $a f$ are not necessarily the geometrical values of the specimens: it is important that they should be chosen in a zone far from transient effects, where it is reasonable to consider CTOA almost constant and equal to its critical value.

Considering that, for the values of angles experienced in the DWT Test, $\operatorname{tg}\left(\alpha_{p}\right) \cdot \operatorname{tg}(\mathrm{CTOA} /$ $2) \ll 1$, the mean value of $\mathrm{CTOA}_{C}$ can be calculated by rearranging Equation 5 :

$$
\overline{\mathrm{CTOA}}_{\mathrm{C}}=2 \cdot \operatorname{arctg}\left\{\frac{r^{*} \cdot E_{P}^{a i \rightarrow a f}}{2 A^{*} \sigma_{0} B \cdot\left[W(a f-a i)-\frac{1}{2}\left(a f^{2}-a i^{2}\right)\right]}\right\}
$$

Now all tools for managing experimental results and drawing the load force-crack length diagram are available. 


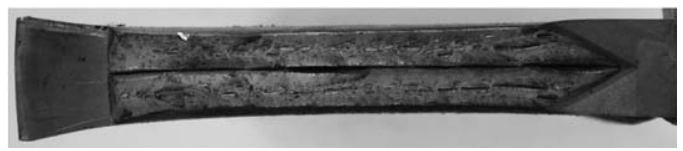

Figure 2. Fracture surface: for DWTT-02.

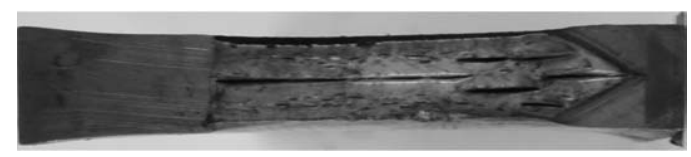

Figure 3. Fracture surface: for DWTT-03.

Table 1. Drop weight tear test specimen characteristics.

\begin{tabular}{lcclc}
\hline Specimen & $\begin{array}{l}\text { Depth notch }\left(a_{0}\right) \\
(\mathrm{mm})\end{array}$ & $\begin{array}{l}\text { Width } \\
(\mathrm{mm})\end{array}$ & $\begin{array}{l}\text { Actual Thickness } \\
(\mathrm{mm})\end{array}$ & $\begin{array}{l}\text { Depth of Back slot } \\
(\mathrm{mm})\end{array}$ \\
\hline DWTT-01 & 9.92 & 76.16 & 12.41 & - \\
DWTT-02 (Chevron) & 10.20 & 76.01 & 11.49 & 11.81 \\
DWTT-03 (Chevron) & 10.20 & 76.01 & 11.29 & 23.94 \\
\hline
\end{tabular}

\section{Tuning of fracture parameters through analysis of experimental data}

The specimens were extracted from a pipeline built of an X80 steel, and then flattened. Two of them were chevron-notched and back slotted, whose fracture surfaces, after a DWT Test, are reported in Figures 2 and 3.

The main characteristics of the specimens (three samples for each type) are reported in Table 1.

As mentioned above, before starting FE simulation it is necessary to evaluate the $\mathrm{CTOA}_{\mathrm{C}}$. A preliminary rough estimate can be carried out considering only the initial crack size (corresponding to the depth notch, that is to say $a=a_{0}$ ) and the final crack size (corresponding to having broken all the ligament, that is to say $a_{\text {FIN }}=W$ ) together with the global energy dissipated during impact. In such case Equation 6 can be written as:

$$
\overline{\mathrm{CTOA}}_{\mathrm{C}}=2 \cdot \operatorname{arctg}\left(\frac{r^{*} \cdot E_{P}^{a_{0} \rightarrow W}}{A^{*} \sigma_{0} B \cdot\left(W-a_{0}\right)^{2}}\right)
$$

This estimate of averaged $\mathrm{CTOA}_{\mathrm{C}}$ is only approximate: it is not able to disregard initial and final transient effects but considers specimen rupture as a whole. As a matter of fact, when the crack starts growing, CTOA is very high and then rapidly decreases, during a transition phase, tending to the stable critical value (Corigliano et al., 1999). On the opposite side of the specimen, when the residual ligament become relatively short, CTOA begins to increase as a consequence of the interaction between the plastic zones under the impacting hammer and the one ahead of the crack tip.

These considerations would in principle contrast with the idea to consider the $\mathrm{CTOA}_{\mathrm{C}}$ as a material constant that can be found in the available literature (Martinelli et al., 1996; Wang, 1995; Kanninen et al., 1995); however, the leading point is that CTOA must be accounted for only during stable conditions, so that the question is how to isolate such event.

It is suitable to adequately choose the accounted-for initial and final value of crack length (respectively named $a i$ and $a f$ ) so as to consider only stable propagation (Rudland et al., 2002). An example of this procedure adopted in back slot specimens is shown in Figure 4. 
Table 2. Calculated values of $\overline{\mathrm{CTOA}}_{\mathrm{C}}$ for DWTT-01 specimen.

\begin{tabular}{|c|c|c|c|c|c|c|}
\hline Specimen & $a i$ & af & $\begin{array}{l}E_{P}^{a i \rightarrow a f} \\
\text { model-1 }\end{array}$ & $\begin{array}{l}E_{P}^{a i \rightarrow a f} \\
\text { model-2 }\end{array}$ & $\begin{array}{l}\overline{\mathrm{CTOA}}_{\mathrm{C}} \\
\text { model-1 }\end{array}$ & $\begin{array}{l}\overline{\mathrm{CTOA}}_{\mathrm{C}} \\
\text { model-2}\end{array}$ \\
\hline DWTT-01 & $20.5 \mathrm{~mm}$ & $30.0 \mathrm{~mm}$ & $493.7 \mathrm{~J}$ & $431.9 \mathrm{~J}$ & $7.5^{\circ}$ & $6.5^{\circ}$ \\
\hline
\end{tabular}

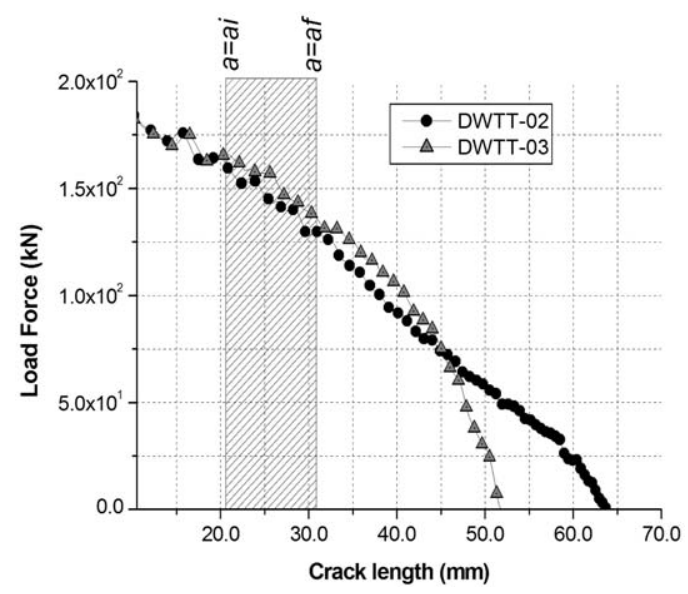

Figure 4. Choice of $a i$ and af values in the stage of stable fracture propagation in the case of DWTT-02 and DWTT-03.

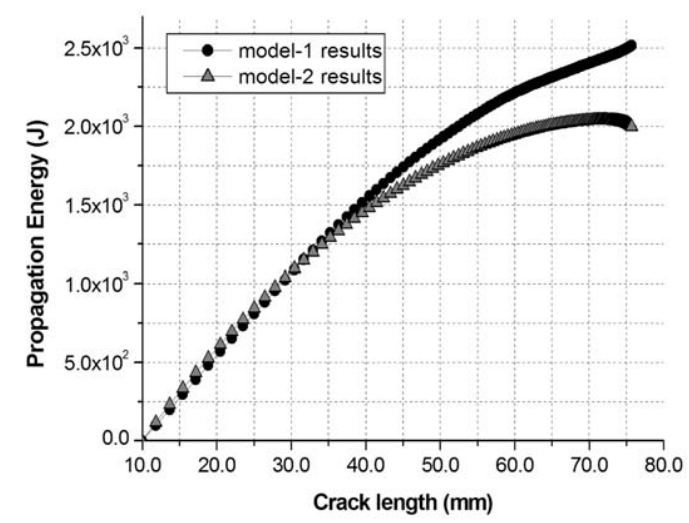

Figure 5. Comparison between model-1 and model-2 propagation energy for DWTT-01.

Suitable values for $a f-a i$ become evident by analysing the scattering present in the followings Figures 5-7, hereinafter discussed. From the point of view of $\mathrm{CTOA}_{\mathrm{C}}$ evaluation, effective extension of $a f-a i$ length can vary moderately.

For DWTT-01 the values of $\overline{\mathrm{CTOA}}_{\mathrm{C}}$ calculated according to this way of proceeding are reported in Table 2.

In Table 2, model-1 indicates the value of energy calculated as the work done (and experimentally measured) by the hammer, corresponding to initial and final crack length (determined by means of kinematic model). On the other hand the model-2 energy represents the same quantity calculated by means of eq. 5 , assuming a constant value of $\mathrm{CTOA}_{\mathrm{C}}$, without making any reference to experimental values; in both cases, attention is focussed also on crack effective total advance, corresponding to $(a f-a i)$.

Values of $\overline{\mathrm{CTOA}}_{\mathrm{C}}$ deduced by model-1 and model-2 energies have been calculated for the three types of specimens, by using eq. 6 . The results show a good accordance between the two methodologies. Differences in model-1 and model-2 energies are mainly a consequence of considering $\mathrm{CTOA}_{\mathrm{C}}$ constant during the propagation process, including initial steps. From this point of view, an initial gap is unrecoverable since energy is always summed during advance. In Figure 5 the graphical comparison between model- 1 and model-2 energies is highlighted.

Attention is now focussed on back slot specimens: Chevron Notched Back Slot DWT Tests (CN-BS-DWTT), which present the important advantage of eliminating, even if not 
Table 3. Calculated values of $\overline{\mathrm{CTOA}}_{\mathrm{C}}$ for DWTT-02 and DWTT- 03 specimens.

\begin{tabular}{|c|c|c|c|c|c|c|}
\hline Specimen & $a i$ & $a f$ & $\begin{array}{l}E_{P}^{a i \rightarrow a f} \\
\text { model-1 }\end{array}$ & $\begin{array}{l}E_{P}^{a i \rightarrow a f} \\
\text { model-2 }\end{array}$ & $\begin{array}{l}\overline{\mathrm{CTOA}}_{\mathrm{C}} \\
\text { model-1 }\end{array}$ & $\begin{array}{l}\overline{\mathrm{CTOA}}_{\mathrm{C}} \\
\text { model-2 }\end{array}$ \\
\hline DWTT-02 & $20.8 \mathrm{~mm}$ & $30.5 \mathrm{~mm}$ & 459.4 J & $441.7 \mathrm{~J}$ & $7.4^{\circ}$ & $7.1^{\circ}$ \\
\hline DWTT-03 & $20.9 \mathrm{~mm}$ & $30.3 \mathrm{~mm}$ & $405.4 \mathrm{~J}$ & $362.7 \mathrm{~J}$ & $6.9^{\circ}$ & $6.1^{\circ}$ \\
\hline
\end{tabular}

completely, the transient effects during the final stages of crack propagation, that is to say, when the residual ligament is too short.

As a matter of fact, in BS-DWTT a slot of material, along the crack plane, is extracted from the back side of the specimen and replaced with a high-strength steel shim (Figure 1) able to guarantee the same boundary conditions as a standard DWTT for, at least, a great part of the crack path. This enhanced specimen allows a crack length, at most, equal to ( $\left.W-a_{0}-L_{B S}\right)$, where $L_{B S}$ represents the slot depth. The Chevron Notch limits initiation energy.

The same procedure, applied before, has been carried out for DWTT-02 and DWTT-03 specimens, giving the results for $\overline{\mathrm{CTOA}}_{\mathrm{C}}$ shown in Table 3 .

Some differences in the value of $\overline{\mathrm{CTOA}}_{\mathrm{C}}$ with respect to those obtained for DWTT-01 are also due to the use of a kinematic model based on the hypothesis of CTOA constancy since the initial stages. Due to the Chevron notch adopted for the specimen, the initiation energy is here intrinsically lower than in previous specimens, therefore the CTOA value (which is an average) is less shifted to higher values by initiation effects and closer to the stable propagating value.

Moreover, an additional problem of the procedure adopted is to find a value of $\overline{\mathrm{CTOA}}_{\mathrm{C}}$ giving an excellent accordance between energy results, but also taking in mind that fracture must propagate for all ligament length. As a matter of fact, an increase of $\overline{\mathrm{CTOA}}_{\mathrm{C}}$ causes a better agreement from an energy point of view, but also, at the same time, a non-completely fractured ligament. In the job carried out, severe trouble has been found in the contemporaneous agreement of the two different aspects. The solution of a very difficult realization consists in evaluating the real trend of CTOA during propagation with a high frame-rate digital camera and extrapolating a mathematical law giving CTOA as a function of crack length.

Hence the value of the crack tip opening angle is probably somewhat lower than that calculated, but it increases before the specimen is completely fractured.

Figures 6 and 7 illustrate the comparison between model- 1 and model- 2 energies and the agreement between numerical and experimental results.

Again as above, the differences of the two energies after a certain crack advance suggest that the phenomenon cannot be explained completely with the kinematic simple assumption.

Something new must also be accounted for in back slot specimens. As a matter of fact, being the specimen subjected to bending, the zone in the back side (that is under the hammer) is compressed, but there is also a part of the sample subjected to traction stress near the crack tip, due to bending resulting forces. The shim can transmit compression but is unable to transmit traction; therefore a wider difference is attended and evidenced. Assuming, for the sake of simplicity, a butterfly stress distribution in the crack plane, it is quite meaningful to consider only propagation until a crack size of approximately $\left(W-a_{0}-2 L_{B S}\right)$, in order to avoid material separation. Keeping in mind the previous remarks, the comparison between 


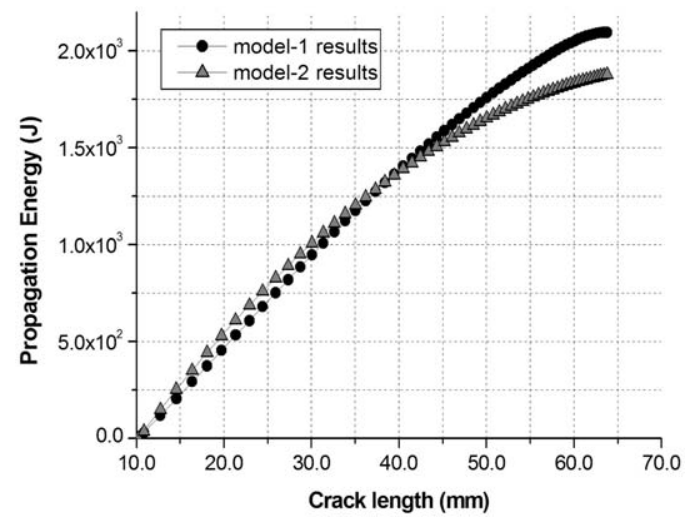

Figure 6. Comparison between model-1 and model-2 propagation energy for DWTT-02.

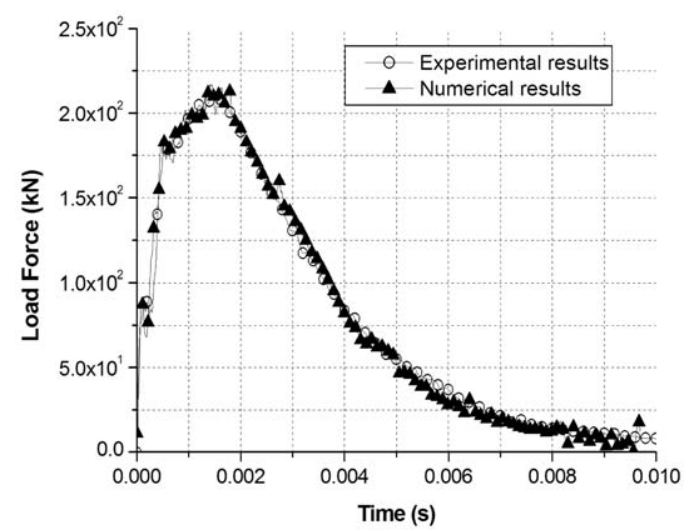

Figure 8. Fitting between experimental and numerical force-time curve for DWTT-01.

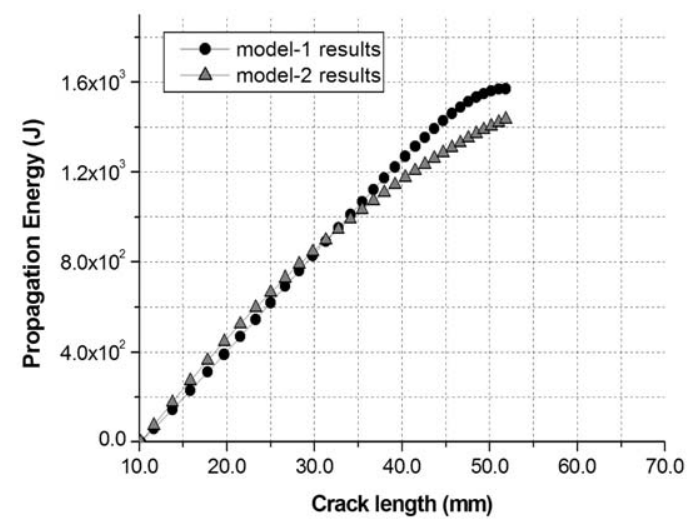

Figure 7. Comparison between model-1 and model-2 propagation energy for DWTT-03.

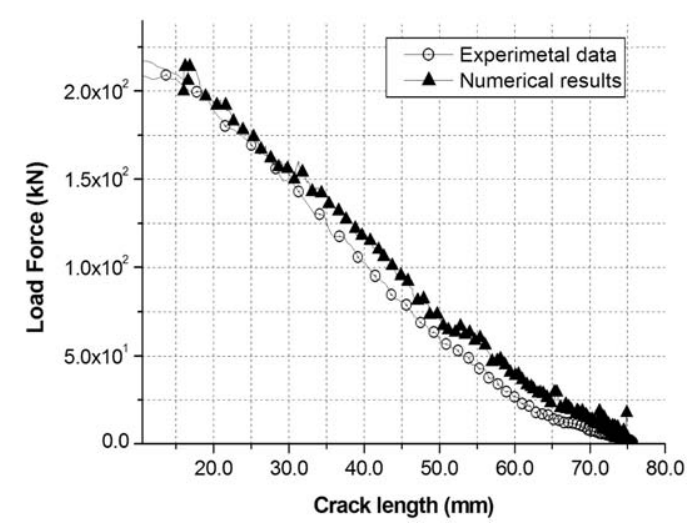

Figure 9. Fitting between experimental and numerical force-crack length curve for DWTT-01.

energy diagrams can now be considered good and leads to similar values of $\mathrm{CTOA}_{\mathrm{C}}$ choosing appropriately the integrated crack path zone.

\section{DWT test simulation by means of finite element analysis}

Once $\overline{\mathrm{CTOA}}_{\mathrm{C}}$ is known, a Finite Element simulation in PICPRO can be started in the attempt to achieve a good accordance between experimental and numerical results. This task can be carried out by varying the size of the process zone $\Delta$ until the numerical load force vs. time curve fits the correspondent experimental one. Fracture initiation point for simulation is always fixed in the correspondence of the maximum load.

As previously mentioned, fracture initiation is not under consideration in this paper. The main aim is to analyse conditions of fracture propagation. PICPRO is designed for simulating crack advance both in stable and transient conditions: crack speed results from the instantaneous comparison of driven force towards critical known values; therefore, the crack tip can 


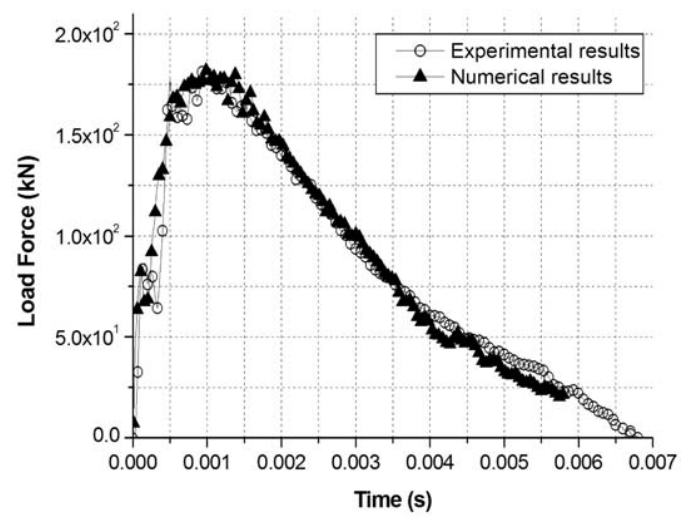

Figure 10. Fitting between experimental and numerical force-time curve for DWTT-02.

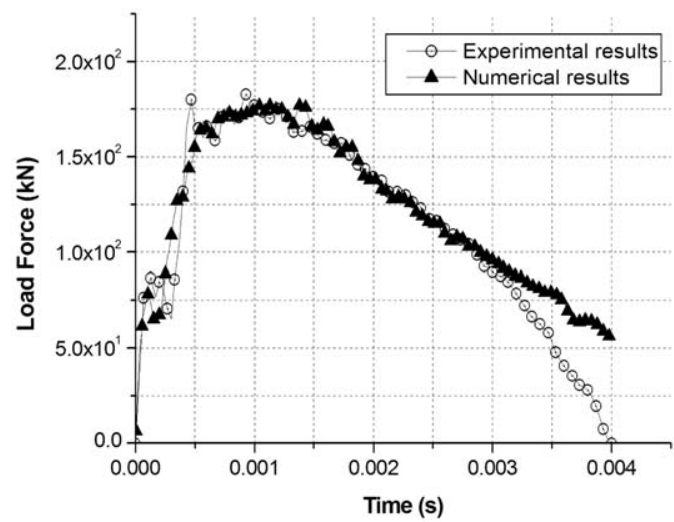

Figure 12. Fitting between experimental and numerical force-time curve for DWTT-03.

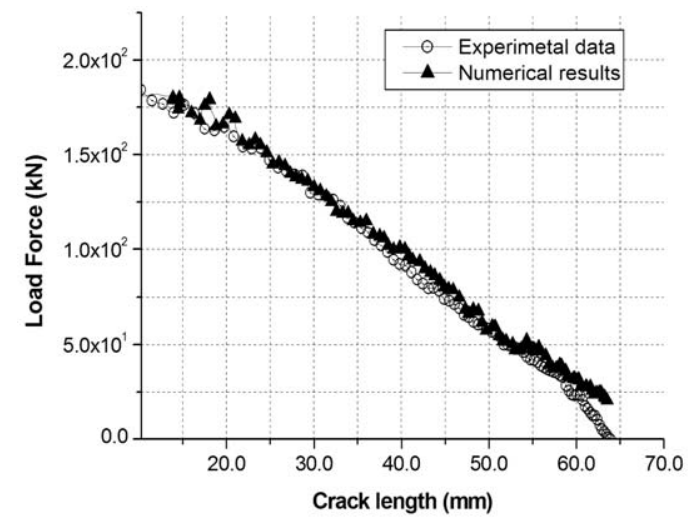

Figure 11. Fitting between experimental and numerical force-crack length curve for DWTT-02.

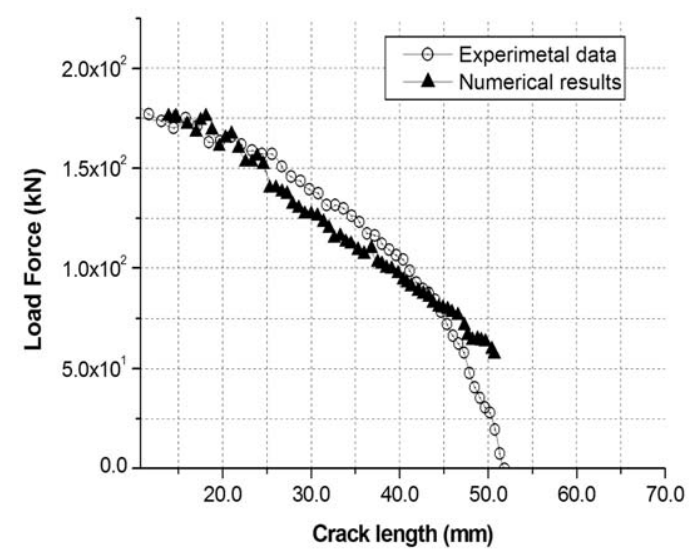

Figure 13. Fitting between experimental and numerical force-crack length curve for DWTT-03.

move at a stable speed, can accelerate or decelerate in the attempt to try to reach new stable conditions of propagation, or, at last, can decelerate until an arrest. Simulation of three DWT Tests has been performed by exclusive use of FPA of PICPRO. For the material and specimens considered here, a good fitting is found by using $\Delta=7.5 \mathrm{~mm}$, corresponding to 0.60 times the thickness.

In Figure 8, comparison between force vs. time curves of DWTT-01 is reported, showing the good fitting of experimental and numerical results reached by FPA, without use of the kinematic model. On the other hand, in Figure 9, the comparison of force vs. crack length curves is shown; a non-excellent fitting can be evidenced due to the approximation of the kinematic model utilized to calculate crack length. Of course, the scatter of two curves is wider after a crack amount, which is in accordance with the results of Figure 5.

From the modelling point of view, the interface between slot shims and specimens is modelled by the adoption of appropriate constraint conditions that prevent compenetration but allow sliding. For back slot specimens, the fitting of force-time curves is good until the final 


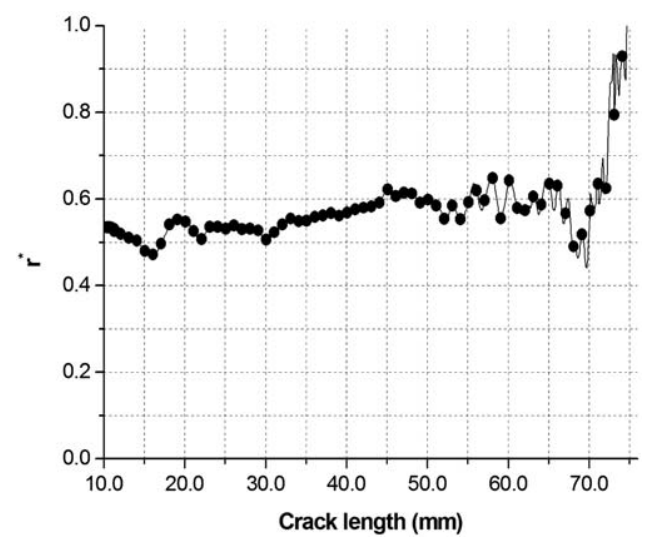

Figure 14. Finite Element simulation: trend of $r^{*}$ parameter in DWTT-01.

part of crack advance (Figures 10,12) where transient effects do occur, due to forced absence of traction stresses mentioned above and to crack tunnelling that causes a lack of constraint when the inner part of the crack reaches the shim (Rudland et al., 2002). Fracture tunnelling is neglected when PICPRO, which makes use of shell elements, is used. Comparisons of experimental and numerical force-crack length curves for back slot specimens are shown in Figures 11, 13.

Numerical simulations evidence also that $r^{*}$ (computed numerically by analyzing the movement of the non-plasticized part of the specimen - Figure 14) increases during the final stage of propagation and is, at last, much higher than the stable critical value (Matsoukas et al., 1984). When applying the kinematic model this value is forcedly kept constant, but if Finite Element analysis is used the value can update each step.

Keeping in mind the simplifications required by the kinematic model and its sensitivity to initial and final approximations, the use of two different slot depths should allow, assuming Equation 5, the evaluation of the propagation energy associated with the extension $(a f-a i)$ equal to the slot depth difference, considering that transient effects have almost the same influence for both lengths.

\section{Conclusions}

In the paper the possible use of back slotted specimens for a DWT Test was discussed.

The idea is promising but, at the present stage, it is difficult to have a precise estimate of the transient part of the propagation energy which affects the precision of the results. Performing DWT tests on different lengths of back slot can give the opportunity to evaluate the extension of the transient part of the energy absorption. Therefore, if results on different materials will confirm, the $\mathrm{CTOA}_{\mathrm{C}}$ could be estimated by use of only global energy results, without adoption of an instrumented hammer. On the contrary, if loads are stored continuously, $\mathrm{CTOA}_{\mathrm{C}}$ can be found with a good precision; in this paper some ways to account for reliability of the results are illustrated. 


\section{References}

Azodi, D. and Kalthoff, J.F. (1990). Evaluation of rapid crack propagation J-values for ductile steels, Proc. of 4th Int. Conf. on Numerical methods in fracture mechanics, Freiburg, Germany, 23rd-27th April 1990.

Berardo, G., Salvini, P. and Di Biagio, M. (2000). Application of cohesive model to dynamic ductile fracture tests, XIII European Conference on Fracture, Elsevier, San Sebastian (Spain).

Corigliano, A., Maier, G. and Mariani, S. (1999). Analysis of ductile fracture in damaged pipelines by a geometric parameter method, Engineering Structures 21, 924-936.

Cotterel, B. and Reddel, J.K. (1977). The essential work of plane stress fracture. Int. J. of Fracture 13, 267-277.

Demofonti, G. et al. (1995). Step by step procedure for the two specimen CTOA test, Proc. of the 2nd International Conference on Pipeline Technology, vol. II, Ostend.

Fonzo, A., Salvini, P., Di Biagio, M. and Mannucci, G. (2002). Full History Pipeline burst test through Finite Element Analysis, Proc. of 4th International Pipeline Conference, Calgary, Alberta, Canada, September 29th - October 3rd 2002.

Kanninen, M.F. and O'Donoghue, P.E. (1995). Research challenges arising from current and potential applications of dynamic fracture mechanics to the integrity of engineering structures, Int. Journal of Solids Structures 32, $2423-2445$.

Knott, J.F. (1973). Fundamentals of Fracture Mechanics, Butterworths, London.

Martinelli, A. and Venzi, S. (1996). Tearing modulus, J-integral, CTOA, and crack profile shape obtained from the load displacement curve only, Engineering Fracture Mechanics 53/2, 263-277.

Matsoukas, G., Cotterel, B. and Mai, Y.M. (1984). On the plastic rotation constant used in standard COD tests, International Journal of Fracture 26, R49-R53.

Newman, J.C. Jr., James, M.A. and Zerbst, U. (2003). A review of the CTOA/CTOD fracture criterion, Engineering Fracture Mechanics 70/3-4, 371-385.

Salvini, P., Berardo, G., Demofonti, G. and Mannucci, G. (1999). A Fracture Process Zone model for CTOA analysis. Int. Conf. On Fracture Damage Mechanics 1999, Queen Mary \& West field College University of London, London (UK), 27-29 July 1999.

Salvini, P., Berardo, G., Demofonti, G. and Mannucci, G. (2000). Numerical model of backfill constraint effect during crack propagation on buried large diameter gas pipelines, Int. Pipeline Technology Conference, I, 315 329, Brugge (Belgium), May 2000.

Salvini, P., Fonzo, A. and Mannucci, G. (2003). Identification of CTOA and Fracture Process Parameters by Drop Weight Test and Finite Element Simulation, Engineering Fracture Mechanics 70/3-4, 553-566.

Rice, J.R. and Sorensen, E.P. (1978). Continuing crack-tip deformation and fracture for plane-strain crack growth in elastic-plastic solids, Journ. Mech. Phys. Solids 26, 163-186.

Rudland, D.L., Wilkowski, G., Wang, Y., Horsley, D., Rothwell, B. and Glover, A. (2002). Investigation into the use of a single specimen for the determination of dynamic steady state propagation resistance in high toughness line-pipe steels, Proc. of 4th International Pipeline Conference, Calgary, Alberta, Canada, September 29thOctober 3rd 2002.

Wang, B., Yang, W. and Hwang, K.C. (1994). An engineering fracture parameter for non-J-controlled crack growth, Fatigue Fract. Engng. Mater. Struct. 17/4, 469-477.

Wang, G.S. (1995). An EPFM analysis of crack initiation, stable growth and instability, Engineering Fracture Mechanics 50, 261-282. 